\title{
Short communications / Kleine Mitteilungen Petites communications / Brevi communicazioni
}

\section{Landarzt vor 300 Jahren}

Der luzernische Chirurg und Geburtshelfer Klaus Melchior Bründler († 1721)

von Joseph Schürmann

ZUSAMMENFASSUNG

Regierungsbeschlüsse und Gerichtsakten enthalten Anhaltspunkte darüber, wie ein "Chirurg» vor 300 Jahren ausgebildet wurde, und welche Leistungen er draussen auf dem Land zu erbringen hatte, zumal bei schwierigen Geburten.

In Totenregistern, von geistlicher Hand aufgezeichnet, finden wir Einzelheiten darüber, wie es einst bei schwierigen, verzögerten Geburten zuging ${ }^{7}$ : «in porrecta manu ab obstetrice baptizatus» (auf dem vorgefallenen Händchen wurde der Knabe von der Hebamme getauft, 1772); «ad caput usque nata a chirurgo baptizata» (erst bis zum Köpfchen geboren, wurde das Mädchen vom Chirurgen getauft, 1783). «Chirurg» war damals «der Handanleger», in Lehrzeit und auf Wanderschaft ausgebildet, der Bruchschneider und Wundbehandler, draussen auf dem Land eben auch «Doktor» genannt, weil notgedrungen auch bei inneren Krankheiten sein Rat und seine Hilfe verlangt wurden.

Über das «Turmbuch» des Staates Luzern - die Einvernahmeprotokolle Angeschuldigter - und über die Ratsbücher mit den Urteilen am Ende der Prozesse vernehmen wir weitere Einzelheiten über abnorme Geburtsverläufe aus dem Munde der Hauptbeteiligten. Wir «Zivilisierten» am Ende des 20. Jahrhunderts können uns ja gar nicht mehr vorstellen, welche Szenen sich da in den niedrigen Kammern von Bauernhäusern abspielten, bei Kerzenlicht, ohne wirksame Schmerz- oder gar Betäubungsmittel, unter dem hilflos-angstvollen Beten und Wehklagen von Angehörigen und neugierigen Nachbarinnen.

Klaus Melchior Bründler entstammte einer Luzerner Bauernfamilie. 
Wann er geboren wurde, müssen wir zu erraten suchen. 1673 habe er die Lehre angetreten, aber just für die Jahre zwischen 1653 und 1659 weist das für den Wohnort seiner Eltern zuständige Taufbuch von Root eine Lücke auf. Die Lehre - der Vater soll dafür 150 Taler ausgelegt haben - durchlief er beim Bruchschneider Johannes Trutmann in Küssnacht, auf Schwyzer Ge-

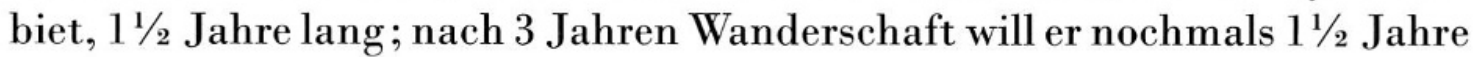
mit Trutmann zusammengearbeitet haben. Sein Lehrbrief sei in Arth, ebenfalls auf Schwyzer Gebiet, ausgestellt worden. Dass solches nicht in der engeren Heimat, in oder um Luzern, geschehen war, sollte ihm später zum Nachteil gereichen. Der Satz «Sey in Wien gelegen, da der Türk es belägeret», weist darauf hin, wie weit damals eine Chirurgen-Wanderschaft ausgedehnt werden konnte. Der Aufenthalt in Österreich hätte demnach 1683, zehn Jahre nach Antritt der Lehre, stattgefunden.

1701 schliesst Bründler in Ruswil die Ehe mit der aus Willisau stammenden Anna Maria Zeder. Bis 1715 schenkt die Frau sechs Kindern das Leben; das erste und das dritte, das zweite und das fünfte tragen je den gleichen Taufnamen, so dass wir annehmen müssen, es hätten bestenfalls vier von den sechsen die ersten Jahre überlebt.

Die erste Notiz über Klaus Melchior Bründlers Wirken als «Chirurg» entnehmen wir dem Eintrag im Ratsbuch vom 30. September 1707' : Es sind Klagen über ihn eingegangen, dass «er sich der Chirurgie annehme, so er doch niemahlen erlehrnet», er habe «2 Weyber in der Kindbetten also vernachlässiget, dass aus seiner Schuld und Ungeschicklichkeit halber die eine gestorben, die andere aber grossen Schaden erlitten.»

Am 26. Oktober 1707 beschliesst der Rat ${ }^{2}$, Bründler solle «betreffend sein Artzten und Assistieren in Kindsnöthen constituiert und von dem Collegio medico examiniert werden.» Drei Tage später, am 29. Oktober $1707^{3}$, hat der Rat seinen Beschluss dahin geändert, dass Bründler «seines Artznens wegen von Herrn Rathsrichtern examiniert werde, mithin ihme solches der Leuten wegen völlig untersagt seye». («der Leuten wegen» deutet wohl an, dass Bründler auch krankes Vieh behandelte.) Über dieses «Examen» durch den Ratsrichter, der Untersuchungsrichter und Staatsanwalt in einer Person war, gibt das Turmbuch ${ }^{4}$ folgende Einzelheiten.

Er hat gelernt: «Bruchschniden, dann Schäden und Fistlen und Wassersucht zu heilen». Eine von ihm (wegen Wassersucht?) behandelte Frau lebe noch und habe seither ein Kind geboren. Er gibt Medizinen ab, habe Wassersucht, die fallende Sucht und andere Schäden geheilt und viele Schäden angenommen, von welchen die Doctores und Chirurgi abgestanden. 
Er habe über tausend Kunden, darunter einen Mann, der von unten herauf ganz verdorrt war und über 150 Taler ausgelegt hatte, und dem niemand habe helfen können. Als Beispiel für «die fallende Sucht» erwähnt er die Tochter eines Kilchmeiers, die täglich zwei Anfälle gehabt hätte und davon ganz befreit worden sei.

Ausführlich wird dann Bründler über sein «Assistiren in Kindsnöthen» befragt. Während seiner Lehre und seinem Aufenthalt bei Trutmann habe dieser 25 Kinder «ausgeschnitten», er, Bründler «etwelche, aber nur solche, die ein Ärmchen herausgestreckt hatten, das brandschwarz und tot gewesen sei, habe solches nie gemacht, ohne dass die Hebamme dabei gewesen wäre und der Pfarrer seine Erlaubnis erteilt hätte, nie auch, die Frauen wären denn schon 〈verschetzt» gewesen.» Wie ist dieses «verschetzt» zu deuten? Ist damit gemeint, dass die Frauen als hoffnungslos beurteilt, ja mit den Sterbesakramenten versehen gewesen wären? Oder dass sich Hebamme und Chirurg darüber einig waren, dass ohne Eingriff mit dem Überleben der Frau nicht zu rechnen sei?

Todesfälle unter solchen Umständen gibt Bründler drei zu; die eine Frau sei nach vier, eine andere nach sechs Wochen gestorben, «der Mutterfluss habe sie verderbt».

«Wie manche Frau noch lebe, von der er ein Kind geschnitten habe», will der Richter wissen. Allein in der Umgebung von Ruswil lebten noch zehn.

«Wie manches Kind er ungefähr ausgeschnitten?» Um $40 \ldots$, und 80 «kehrt» (äussere oder gar innere Wendung?). Er ruft Zeugen nicht nur aus dem Luzernbiet sondern auch aus Uri und Unterwalden an.

Seine Instrumente habe er in Zug von einem jungen Büchsenschmied anfertigen lassen; als Vorlagen hätten Instrumente seines Lehrmeisters gedient.

Am Ende dieser Einvernahme vom 29. Oktober bittet der Angeschuldigte um Schutz der Regierung vor Leuten, die ihm übel wollen, und «so er nit Doctoren könne, müsse er an ein ander Ort mit Weib und Kind [...] die Leute wüssten, was er könne und liefen ihm Tag und Nacht nach».

Am 3. November 1707 verlangt der Rat ${ }^{5}$, dass Bründler seinen Lehrbrief dem Collegio medico vorlege und dass «Information seiner Curen halber aufgenommen werde».

Protokolle des Collegium medicum aus dieser Zeit liegen nicht vor. Die Frage der Berufserlaubnis wurde auch vom Rat direkt nicht weiter behandelt. So wissen wir nicht, wie der Lehrbrief gelautet hat, und wie er beurteilt worden ist. Und doch enthält das Ratsbuch eine grosse Überraschung: Am 
12.Dezember 1707 lässt ein Mann aus Ruswil «in aller Untertänigkeit» vortragen, sein Bruder befinde sich in sehr elendem Zustand und könne von niemand kuriert werden. Klaus Melchior Bründler habe sich anerboten, ihn um 10 Taler zu heilen, und falls das nicht gelinge, werde er keinen Pfennig fordern. Der Bittsteller ersucht die Gnädigen Herren, dem Bründler die Behandlung zu gestatten, und die Hohe Obrigkeit sagt das denn auch «in Gnaden» zu.

Sieht es nicht fast so aus, als habe Bründler einen Patienten vorgeschickt, um seine Berufsausübung von den Gnädigen Herren bestätigt zu bekommen? Ganz reizvoll ist erst noch der Hinweis auf ein «Erfolgshonorar».

Klaus Melchior Bründler ist am 21. Mai 1721 in Ruswil gestorben. 


\section{Quellen und Literatur}

1 Staatsarchiv Luzern RP $8843 \mathrm{v}$.

2 Staatsarchiv Luzern RP $8851 \mathrm{v}$.

3 Staatsarchiv Luzern RP $8852 \mathrm{v}$.

4 Staatsarchiv Luzern cod $462081 \mathrm{r}$.

5 Staatsarchiv Luzern RP 88 54r.

6 Staatsarchiv Luzern RP 88 60r.

7 Schürmann, Joseph, Medizinisches aus Luzerner Sterbebüchern, in: Gesnerus 45 (1988), $543-560$.

\section{Summary}

Rural medicine in the Canton of Lucerne 300 years ago:

Klaus Melchior Bründler († 1721)

The vocational training of a surgeon 300 years ago and his working within the rural surroundings, especially in obstetrics, are described on the basis of decisions and measures taken by authorities and courts.

\section{Résumé}

Un médecin de campagne d'il y a 300 ans : le Lucernois

Klaus Melchior Bründler († 1721)

La formation et l'activité de ce chirurgien et obstétricien sont discutées d'après des documents judiciaires et gouvernementaux.

Dr. med. Joseph Schürmann

Seefeldstr. 6

CH-6006 Luzern 


\section{Prix et Médaille Marc-Auguste Pictet 1992}

La Société de Physique et d'Histoire naturelle de Genève a décerné le Prix M.-A. Pictet 1992 à Diana L.Barkan pour son travail intitulé «Walther Nernst and the transition to modern physical chemistry». Madame Barkan est professeur d'histoire des sciences au California Institute of Technology de Pasadena. Le prix lui a été remis le 22 avril 1992 à Genève.

Lors de la même cérémonie, la Médaille M.-A. Pictet fut présentée à George B. Kauffman, professeur de chimie à l'Université de l'Etat de California à Fresno, pour honorer un des meilleurs connaisseurs d'Alfred Werner. Werner, né à Mulhouse en Alsace en 1866, fut professeur de chimie à l'Université de Zurich de 1893 jusqu'à sa mort en 1919. Il créa la theorie de coordination chimique et reçut en 1913 le Prix Nobel. Lors de la remise de la Médaille Pictet, le Professeur Kauffman donna une conférence qu'il a résumée ainsi :

\section{Alfred Werner, the first Swiss Nobel Chemistry Laureate}

Alfred Werner was the undisputed founder and systematizer of coordination chemistry. He devoted his scientific career to amassing the experimental evidence required to prove the validity of his coordination theory, which he proposed in 1893 at the age of 26 . Beginning with a study of the hitherto unexplained "molecular compounds" (metal-ammines, hydrates and double salts), his ideas soon encompassed almost the whole of systematic inorganic chemistry and even found application in the organic realm. He was the first to demonstrate that stereochemistry is a general phenomenon not limited to carbon compounds and even today his experimental and theoretical papers remain a foundation and guide for investigations in coordination chemistry. In 1913 he was awarded the Nobel Prize in chemistry in recognition of "his work on the linkage of atoms in molecules, by which he has thrown fresh light on old problems and opened new fields of research, particularly in inorganic chemistry".

This lecture, featuring numerous slides, reviews the life and work of the man known as "the inorganic Kekulé". 


\section{Centro Tedesco di Studi Veneziani}

\section{Das Deutsche Studienzentrum in Venedig}

Ein Forschungsinstitut für Postgraduierte und jüngere Wissenschaftler

Das Deutsche Studienzentrum in Venedig ist unter den wissenschaftlichen Instituten, die der deutsche Staat in Italien unterhält, das jüngste. Im November 1990 wurde mit einem Festakt das zwanzigjährige Bestehen gefeiert. Der Chemiker und Nobelpreisträger Adolf Butenandt, der Byzantinist Hans-Georg Beck, der Nationalökonom Alexander Kreuter, der Rechtshistoriker Helmut Coing sowie die Kunsthistoriker Erich Steingräber und Wolfgang Wolters hatten seit 1964 die Idee der Gründung eines Instituts in Venedig verfochten, das wissenschaftliches Arbeiten zur Kulturgeschichte des Veneto bzw. Oberitaliens vor Ort ermöglichen sollte. 1969 wurde hierfür der Palazzo Barbarigo della Terrazza am Canal Grande mit Hilfe der FritzThyssen-Stiftung erworben und restauriert. Eine entsprechende Initiative der deutschen Bundesregierung und ein Beschluss des Bundestages sicherten die Übernahme der Folgelasten sowie der Stipendien. Sieben Universitätsprofessoren bilden seither das wissenschaftliche Kuratorium, das u.a. über die Forschungs- bzw. Stipendienanträge entscheidet. Im Frühjahr 1972 konnten die ersten Stipendiaten ihre Unterkünfte beziehen. Erster Direktor wurde der heutige Ordinarius für Kunstgeschichte in Berlin, Prof. Wolfgang Wolters. Nach einem Historiker (Hans-Eberhard Mayer), einem Italianisten (Bodo Guthmüller), einem Philosophie-Historiker (Eckhard Kessler), einer Rechtshistorikerin (Karin Nehlsen-v. Stryk) sowie einem weiteren Historiker (Bernd Roeck) steht seit Oktober 1990 mit Priv. Doz. Dr. med. Dr. phil. Klaus Bergdolt erstmals ein Medizinhistoriker dem Institut vor.

Der Centro Tedesco di Studi Veneziani bietet sieben bis acht Stipendiaten Forschungsmöglichkeiten. Stipendien werden in der Regel an graduierte Wissenschaftler vergeben, die, sofern der Arbeitsschwerpunkt Venedig bzw. Oberitalien betrifft, hier ihre Promotion oder Habilitation fortsetzen können. In der Regel umfasst die Aufenthaltsdauer, je nach Thema, zwei Monate bis zwei Jahre. Das Stipendium schliesst eine grosszügige Finanzierung sowie freie Unterkunft ein. Seit 1979 werden in beschränkter Anzahl auch Kurzstipendien an Künstler vergeben. Entsprechende Anträge (zwei Gutachten von Hochschullehrern, Sprachnachweis sowie die üblichen Unterlagen) sind an den derzeitigen Präsidenten des Kuratoriums, Prof. Dr. Dr.h.c. Dieter Nörr, Professor-Huber-Platz 2, 8000 München 22, zu richten, im Falle eines Künstlerstipendiums an das Bundesinnenministerium, Post- 
fach 1702 90, 5300 Bonn 1. Allgemein werden Stipendien nur an deutsche Staatsbürger vergeben. Die Benutzung der Bibliothek steht dagegen - nach Antrag an den Direktor - auch Nichtstipendiaten und Angehörigen anderer Länder frei, sofern venezianische Forschungsthemen bearbeitet werden.

Das Institut veranstaltet ein- bis zweimal monatlich wissenschaftliche Vorträge mit internationalen Referenten sowie -in der Regel einmal jährlich - einen internationalen wissenschaftlichen Kongress. Der Direktor ist Herausgeber einer Heftreihe "Quaderni» sowie einer Buchreihe «Studi», die gemäss dem interdisziplinären Charakter des Hauses verschiedene Themen behandeln und namentlich Beiträge hier durchgeführter Kongresse wiedergeben.

Angesichts der überragenden Bedeutung Italiens für die europäische Medizin- bzw. Naturwissenschaftsgeschichte ist zu hoffen, dass künftig auch Historiker dieser Fachrichtungen die Möglichkeiten des «Centro Tedesco» in Venedig vermehrt nutzen werden.

PD Dr. med. Dr. phil. Klaus Bergdolt

Centro Tedesco di Studi Veneziani

Palazzo Barbarigo della Terrazza

S. Polo 2765 a, I-30125 Venezia 